\title{
REVIEW
}

\section{The Promise of E-Platform Technology in Medical Education}

\author{
Siraj Dawd ${ }^{1}$
}

\section{ABSTRACT}

Increasing the number as well as improving the capacity and quality of medical professionals to achieve an equitable health care for all is a global priority and a global challenge. In developing countries, which are facing the largest burden of disease, to achieve the above stated objective, there is a big need for more well-trained, competent and dedicated health care providers. Currently, there is a well-documented shortage of trained health workers globally, with the poorest countries having the greatest shortfalls.

The time tested, traditional approach of training health care force by importing professionals from overseas is not only prohibitively expensive but also not sufficient to achieve the scale and pace of the required human capacity building. Considering this fact, distance learning programs, which include mHealth as well as other information technology (IT) platforms and tools, can provide unique, timely, costeffective, easily scalable and valuable opportunities to expand access to training health care manpower in developing countries where the shortage is critical.

KEYWORDS: E-Platform technology, Vedio conference, Distance learning

DOI:http://dx.doi.org/10.4314/ejhs.v26i2.11

\section{INTRODUCTION}

Increasing the number, improving the capacity and quality of medical professionals to achieve an equitable healthcare for all is a global priority and a global challenge. In developing countries like Africa, which are facing the largest burden of diseases, to achieve the above stated objective, there is a big need for more well-trained, competent, and dedicated health care providers. Currently, there is a well-documented shortage of trained health workers globally, with the poorest countries having the greatest shortfalls. The World Health Report for 2006 estimated that there was a global shortfall of at least 2.4 million health workers. Fifty-seven countries of which 36 are in Sub-Sahharan Africa have a critical shortfall health workers(1). with the increasing world population and near-stagnant training of manpower, the problem is worsening from year to year (Figure 1) (2).
The Global Health Workforce Alliance was set up in 2006 as a response to the World Health Report of that year by WHO and its partners to advocate for the importance of human resource development, and to identify and spread best practice and the findings of research. It, in turn, created several task forces to review and make recommendations in specific areas. In 2007, it established the Task Force on education and training, which was charged with identifying how to accelerate massively the education and training of health workers in those countries in crisis (3).

The conclusions of this task force clearly states that meeting these pressing needs for human capacity building in health care and research requires additional resources and innovation. Traditional approaches to train manpower resource needed to fill the existing gap and future shortfalls are important and necessary but not sufficient to achieve the scale and pace of human capacity building required (4). Considering this

DOI: http://dx.doi.org/10.4314/ejhs.v26i2.10

\footnotetext{
${ }^{1}$ Brody School of Medicine Doctors, North Carolina, United States

Corresponding Author: Siraj Dawd, Email: SIRAJD@ecu.edu
} 
fact, distance learning programs, which include mHealth as well as other information technology (IT) platforms and tools, can provide unique, timely, cost-effective, easily scalable and valuable opportunities to expand access to training healthcare manpower in developing countries where the shortage is critical (5).

\section{Information Technology and E-Platform Learning}

E-learning, which is defined as learning and teaching online through network technologies, is arguably one of the most powerful responses to the growing need for education (6). E-platform distance learning involves the strategic use of multiple IT tools in the learning process. Many studies have reviewed the outcome of e-learning as a mode of teaching including in medical education. Despite the relatively recent introduction of e-learning, those studies reveal no significant difference in learning outcomes, commonly measured as grades or exam results, between traditional and e-learning modes of delivery (7).

E-platform distance learning can be done either synchronously or asynchronously. Synchronous e-learning requires simultaneous participation of all learners and instructors at different locations. Asynchronous e-learning does not require simultaneous participation of learners and instructors. There are advantages and disadvantages to adopting either a synchronous or asynchronous platform. Synchronous e-learning is a real time communication of the two participants, and it is commonly supported by media such as videoconferencing and chat. For this reason, this mode of communication has a potential to be near the real traditional learning process and tends to be more social and avoids frustration by asking and answering questions in real time (8). Synchronous sessions help e-learners feel like participants rather than isolates. Despite those benefits, synchronous learning has major drawbacks related to the availability and predictability of broadband width in developing countries. This lack of predictable broadband access has a potential to derail and frustrate participants because of frequent unanticipated interruptions and poor quality transfer of information. Additionally, the time zone difference between different areas of the world technically limits its practical application.
On the other hand, Asynchronous e-learning, commonly facilitated by media such as e-mail, downloadable teaching materials, interactive tutorials posted on the internet and discussion boards supports communication even when participants cannot be online at the same time. Its key advantage is the flexibility of the media. Asynchronous e-learning makes it possible for learners to $\log$ on to an e-learning environment at any time and download documents or send messages to teachers or peers. In developing countries of Africa, where E-platform learning is considered, asynchronous mode of teaching minimizes the impact of limited internet access. Students may spend more time refining their contributions, which are generally considered more thoughtful compared to synchronous communication (9). The main limitation of asynchronous e-learning is the absence of direct, face-to-face and engaging communication between the learner and the teacher. This can potentially derail the major benefit of teaching which is direct communication and feedback.

An essential challenge thus when choosing the right mode of e-learning in developing countries is to critically study the benefits and limitations of emerging types of asynchronous, synchronous and hybrid e-learning as well as the strength and weakness of those technologies with in the given country. This will facilitate understanding of the complex task ahead-taking advantage of emerging media in ways that benefit learning.

\section{Health Systems in Ethiopia}

Ethiopia's healthcare needs are immense and complicated. Located in the Eastern part of Africa traditionally called the "horn of Africa", Ethiopia is home to over 90 million people, making it the second biggest nation in sub Saharan Africa. The population is growing at an alarming pace and is expected to exceed 100 million by 2020 (10). Despite the impressive progress that has been made to improve access to basic healthcare for the population over the last few years (90 per cent of the population has access to the primary healthcare service), infant, child and maternal mortality rates remain high. The infant mortality rate is 47 per 1000 live births; the child mortality rate is 68 per 1000 live births; the maternal death 
rate is 350 per 100,000 live births; and life expectancy at birth is 62 years for male and 65 years for female $(11,12)$. The country has one physician for 56,000 and one nurse for 3000 people. The highest and lowest physician to population ratios in the public health sector were found to be $1: 28,000$ and $1: 118,000$ in 1989 and in 2006 , respectively $(12,13)$. The communicable diseases most associated with poverty have been compounded by diseases of urbanization and economic development, such as cardiovascular diseases and road side trauma, have been compounded by diseases of urbanization and economic development.

The health budget is less than $7 \%$ of the national government budget. The total healthcare facilities available in the entire country include 139 hospitals with 11,685 beds, 635 health centers, 1,206 health stations and 2,264 private clinics (12). Throughout the clinical settings, lack of trained healthcare professional staff remains a major impediment to the improvement of the nation's healthcare system and delivery of optimal patient care. Until 2006, for over 70 million population, there were only three medical schools with a total yearly intake of 300 students. By 2006 , the total graduate output of those three schools combined was only 4,484 physicians (12).This very low yearly graduation of physicians coupled with immigration has depressed the number of practising physicians in the country even further. By 2006, there were only 636 physicians working in the public sector. Considering this, the government has developed a new strategy of "overflowing the market" in hope of training and retaining adequate physicians in the country (12). With this plan, the number of medical schools and thus the intake have increased in a very short time to unprecedented level. Today, there are 25 medical schools with a yearly intake of over 3000 medical students. This rapid and unprecedented surge has created a major strain in the training of those students. Some of the medical schools that are recently opening only have the basic minimum number of faculty to conduct acceptable training of students. Recognizing this, the Mnistry of Health is now advocating the twining of medical schools with western universities to support them with their medical training. Considering the prohibitive cost and logistics of recruiting and relocating medical trainers in such a scale, the Ethiopian Ministry of Health has recognized training support through eplatform system, the most preferable and sustainable method of teaching support to be pursued. With the gradual improvement of internet technologies, the relatively low cost of producing and maintaining of e-platform educational support makes it the most promising method of support in medical training. Now, the next step is to design the right type of E-platform media of learning and test its effectiveness in real time.

\section{East Carolina University and E-platform Learning Experience}

The ECU telemedicine program has been in continuous operation since its inception in 1992, making it one of the longest running clinical telemedicine operations in the world. The Telemedicine Center provides clinical telehealth services and support; conducts telehealth research; consults and oversees new and existing statewide telehealth networks; and openly educates healthcare providers and the public on the utility of telehealth (Figure 2). 


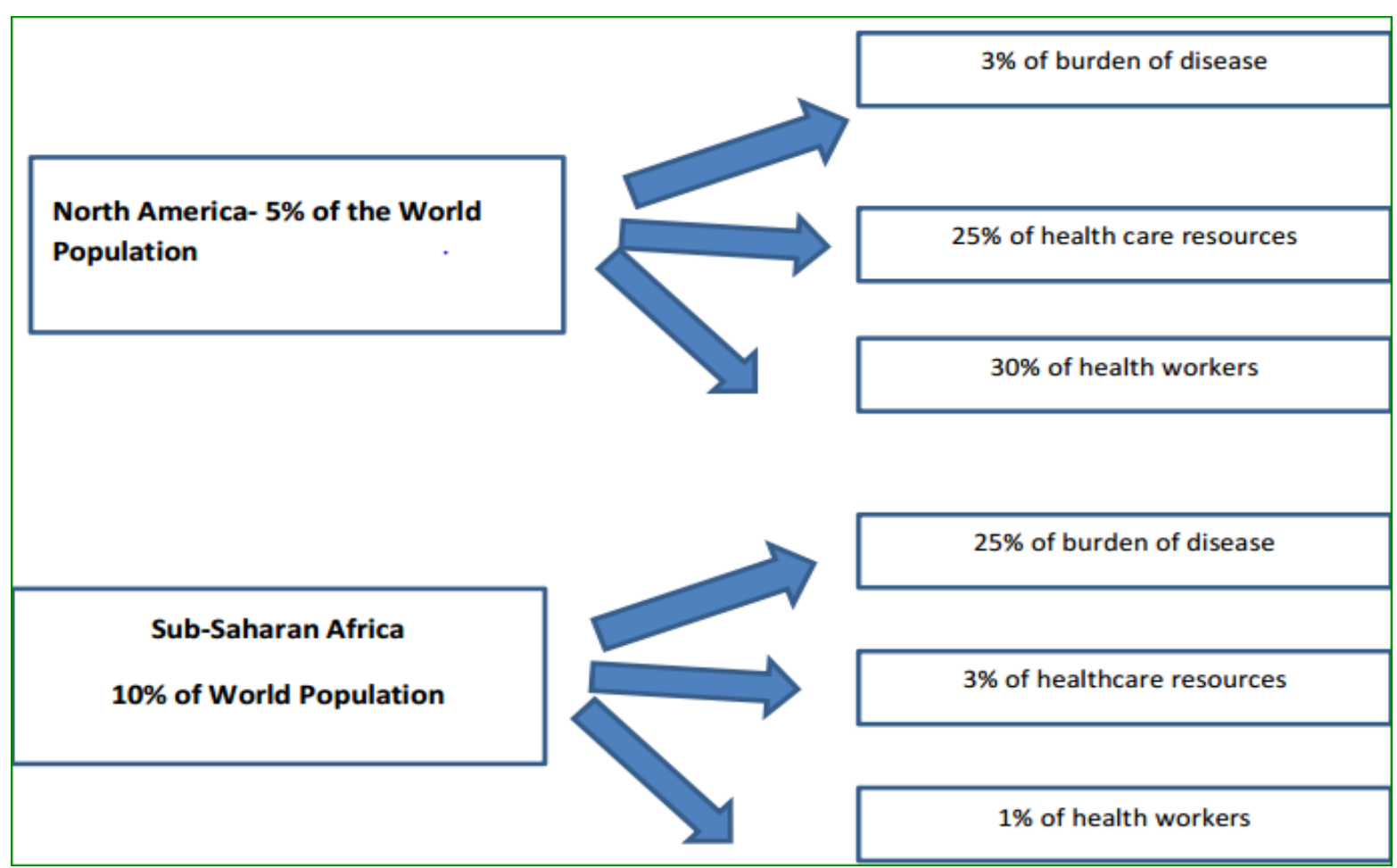

Figure 1: Population resources and burdens in North America and Africa. Modified from Crisp N. Turning the world upside down- the search for global health in the $21^{\text {st }}$ century ${ }^{2}$

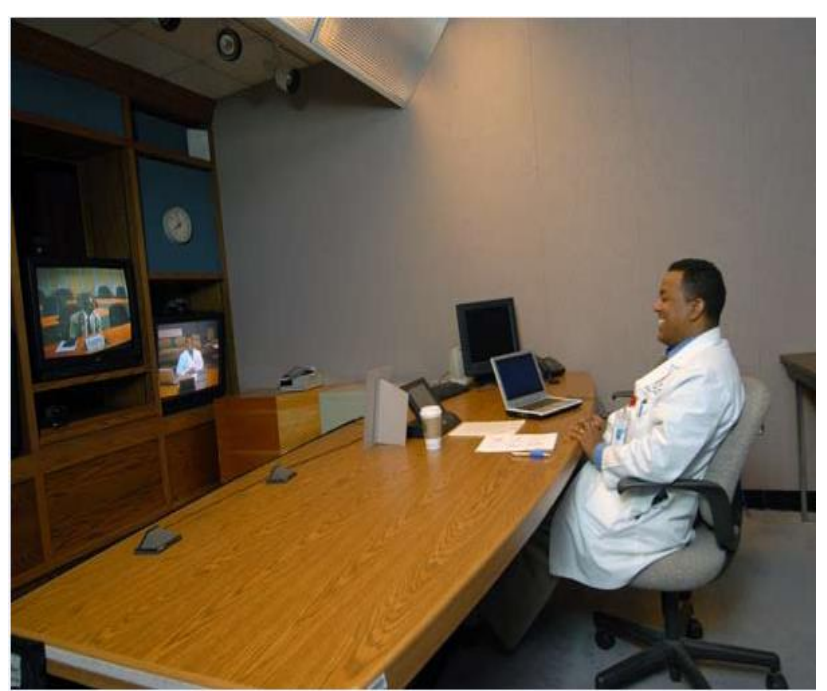

Figure 2: Tele Medicine Forum: East Carolina University connecting with Black Lion Hospital in Addis Ababa, Ethiopia

\section{International Telemedicine}

One of the activities where ECU has been taking part has been the International Tele-Medicine. The forum which was organized by Johns Hopkins Center for Clinical Global Health Education grew

out of the need to provide state of the art clinical guidance regarding the startup and potential sideeffects of HIV antiretroviral therapy (ART) in Ethiopia (14). Clinicians in Ethiopia prepare

difficult HIV cases for bi-monthly presentations to HIV experts based in the United States (Infectious Disease physicians from Johns Hopkins, NIH, Mayo Clinic and East Carolina Universities have been regular participants). Discussions and learning points are simultaneously webcasted so that other providers throughout Ethiopia can participate. All webcasts are also archived for future review and use. Best clinical practices and evidence-based medicine regarding HIV/AIDS care are emphasized. The format has been well received and since its conception, the program has grown remarkably with a visible increase in the number of sites and attendees to the conferences.

\section{Global Understanding Course}

Increasingly, the world is becoming interconnected and medical professionals from 
different countries are working together towards a common goal of global health. This has brought the need to know and understand people, culture and customs of different countries. This, in addition to the actual knowledge gained, breaks the barrier of stigma. This is the basis for the creation of global understanding course.

In a world that is increasingly interconnected, these programs provide first hand, individual, international experiences for the overwhelming majority of our students who cannot study abroad. The global understanding course is taught in a shared virtual classroom with students and faculty at 47 universities, in 28 countries in the Middle East, Africa, Asia, Europe, North and South America (15). Daily video-based dialogs, chats and joint student projects provide students with personal and global experience that opens their prospective about other cultures. These inter-net tools support partner universities anywhere in the world. In giving this course, we use video conference to bring in students from one country for 5 weeks where they learn about each other's culture; students are partnered one-on-one and the two partners have to work on a collaborative project. This process is repeated in the second and third 5-week period with a different country. Thus, at the end of a 16-week semester, our students will have sat in the same class with students from three different countries and known at least one partner student quite well during the collaborative project.

\section{Global Academic Initiatives: Medical Lecture Series}

The Medical Lecture Series is a program that began in 2010 between a few partnering countries and East Carolina University. The program is intended to be a lecture series. There is no cost associated with participation, and there is no ECU course associated with the program. This series consists of non-credit 1-hour sessions offered each Tuesday from 8 Am-9 Am Eastern Standard Time during the months of October - April. The sessions are offered via teleconferencing to partner countries. Participat universities include Moldova University (Moldova), Kufa University (Iraq), Jimma University (Ethopia) and Mekelle University (Ethiopia)

The presentations are led by practising physicians and professors of medicine from East Carolina University's Brody School of Medicine.
ECU Telemedicine has been involved in multiple modalities of E-platform learning to developing countries. Unfortunately, most of those initiatives have been fragmented and based on limited needs assessments. The relationship and connections created with those countries and the bridging faculties involved will be a platform to envision and design the E-platform courses that our university will embark on in the future.

\section{Going Forward}

There is a big manpower demand in the healthcare of developing countries. To fulfill this demand, countries have to train massive numbers of healthcare professionals within a short period of time. To realize this dream, countries have to resort to innovative ideas which have maximum impacts with limited costs. Distance learning programs, which include E-platform as well as other information technology (IT) platforms and tools, can provide unique, timely, cost-effective, easily scalable and valuable opportunities to expand access to training healthcare manpower in developing countries where the shortage is critical (16). The pedagogical approach and choice between synchronous, asynchronous or blended modalities will vary from country to country. Developing countries must recognize the potential of advancing their internet technology and broadband capacity to allow the full development of the E-platform training. At the current level of limited internet access and broadband capability, African countries should blend the training approach to combining web-based, asynchronous, self-paced, distance learning with live, regularly scheduled video conference that augment the asynchronous training and packaged limited faceto-face on-site teaching. The web-based education will be didactic in nature and will introduce the learners to fundamentals of the subject matter. This can be in a form of recorded video clips, pdf files and web-based teaching materials that can be downloaded at the respective institutes and used off line. The video conference live connection and on-site teaching will reinforce the web-based didactic education through discussion of key points as well as through case-based and problembased teaching with emphasis on clinical application.

\section{CONCLUSION}


We are at a cross road in regard to future mode of health manpower training. Developing countries must take E-platform technology seriously and adopt it quickly with modification to their respective countries' needs. They must accelerate the investment in increasing the reach of internet and their broadband capacity. The future will create a fault rift between countries who envisioned and invested in E-platform technology and those who did not. Once allowed to be created, bridging this gap might prove impossible and will cost far more both financially and in human toll in the future.

\section{REFERENCES}

1. World Health Organization. Working together for health. World Health Organization (WHO); May 2006. Last accessed on $22^{\text {nd }}$ of October 2015. http://www.who.int/whr/2006/en/.

2. Crisp N. Turning the world upside down-The search for global health in the $21^{\text {st }}$ century. (London). RSM Press; 2010.p 30.

3. Global Health Workforce Alliance. Scaling up, saving lives. Global Health Workforce Alliance (GHWA); May 2008. Last accessed on $22^{\text {nd }}$ of 2015. http://www.who.int/workforcealliance/docum ents/Global_Health\%20FINAL\%20REPORT. pdf

4. Kinfu H, Dal Poz MR, Mercer H, Evans DB: The health worker shortage in Africa: are enough physicians and nurses being trained? B World Health Organ 2009, 87:225-230.

5. P. L. Beux , M. Fieschi. "Virtual biomedical universities and elearning", International Journal of Medical Informatics, vol. 76, pp. 331- 335, 2007.

6. Zhang DD, Zhao JL, Zhou L, Nunamaker JF Jr. "can E-learning Replace Classroom Learning?" Communications of the ACM, vol.47, no.5 (May 2004), pp.75-79.

7. Bridge PD, Jackson M, Robinson L. The effectiveness of streaming video on medical student learning: a case study. Med Educ
Online 2009;11:1-5. http://www.med-edonline.org

8. Hrastinski S. A Study of asynchronous and synchronous e-learning methods discovered that each supports different purposes. Educause Quarterly, 2008;4; 51-55.

9. Hrastinski S. "the potential of synchronous Communication to Enhance Participation in Online Discussions," paper presented at the $28^{\text {th }}$ International Conference on Information Systems, Montreal, Canada, December 9-12, 2007.

10. Aynalem Aduga. Ethiopian demographic and health: population policy and projection [Internet].Ethiopian Demography and Health. Available from: [Accessed October 12, 2015]. Www.ethiodemographyandhealth.org

11. Federal Democratic Republic of Ethiopia Ministry of Health. Health and health related indicators. Addis Ababa, Ethiopia: Policy Plan Directorate; 2009/10. http://www.nationalplanningcycles.org/sites/d efault/files/country_docs/Ethiopia/ethiopia_hs dp_iv_final_draft_2010_-2015.pdf

12. www.unicef.org/infobycountry/ethiopia_statis tics.html. Ethiopia health statistics. Last accessed October 18, 2015.

13. Berhan Yifru. Medical Doctors Profile in Ethiopia: Production, Attrition \&Retention. In memory of 100 years of Ethiopian Modern Medicine \& the New Ethiopian Millennium. Ethiop Med J. 2008 Jan;46 Suppl 1:1-77.

14. Ethiopia HIV Clinical Case Discussions. Johns Hopkins Center for Clinical Global Health Education. http://main.ccghe.net/casearchive-ethiopia

15. East Carolina University Global Academic Initiatives. Global Understanding Course. http://www.ecu.edu/csacad/globalinitiatives/course.cfm

16. Frehywot S, Yianna Vovides Y, Zohray Talib Z, Nadia Mikhail N, Heather Ross H, Hannah Wohltjen $\mathrm{H}$ et al. E-learning in medical education in resource constrained low- and middle-income countries. Human Resources for Health 2013, 11:4; 1-15. 